\title{
Quantifying direct and indirect contacts for the potential transmission of infection between species using a multilayer contact network
}

\author{
Matthew J. Silk ${ }^{\mathrm{a}, *}$, Julian A. Drewe ${ }^{\mathrm{b}}$, Richard J. Delahay ${ }^{\mathrm{c}}$, Nicola Weber ${ }^{\mathrm{d}}$, \\ Lucy C. Steward ${ }^{\text {a }}$, Jared Wilson-Aggarwal ${ }^{\text {a }}$, Mike Boots ${ }^{\mathrm{d}, \mathrm{e}}$, \\ David J. Hodgson ${ }^{d}$, Darren P. Croft ${ }^{\mathrm{f}}$ and Robbie A. McDonald ${ }^{\mathrm{a}, *}$ \\ ${ }^{\text {a }}$ Environment and Sustainability Institute, University of Exeter, Penryn, Cornwall, UK \\ ${ }^{b}$ The Royal Veterinary College, Hawkshead Lane, North Mymms, Hatfield, Hertfordshire, \\ UK \\ ${ }^{c}$ National Wildlife Management Centre, Animal and Plant Health Agency, Gloucestershire, \\ UK \\ ${ }^{\mathrm{d}}$ Centre for Ecology and Conservation, University of Exeter, Penryn, Cornwall, UK \\ ${ }^{\mathrm{e}}$ Integrative Biology, University of California, Berkeley, CA, USA \\ ${ }^{\mathrm{f}}$ Centre for Research in Animal Behaviour, University of Exeter, Exeter, UK \\ *Corresponding authors' e-mail addresses: matthewsilk@outlook.com, \\ r.mcdonald@exeter.ac.uk
}

Received 23 December 2017; initial decision 15 February 2018; revised 19 March 2018; accepted 12 April 2018; published online 8 May 2018

\begin{abstract}
Detecting opportunities for between-species transmission of pathogens can be challenging, particularly if rare behaviours or environmental transmission are involved. We present a multilayer network framework to quantify transmission potential in multi-host systems, incorporating environmental transmission, by using empirical data on direct and indirect contacts between European badgers Meles meles and domestic cattle. We identify that indirect contacts via the environment at badger latrines on pasture are likely to be important for transmission within badger populations and between badgers and cattle. We also find a positive correlation between the role of individual badgers within the badger social network, and their role in the overall badger-cattle-environment network, suggesting that the same behavioural traits contribute to the role of individual badgers in within- and between-species transmission. These findings have implications for disease management interventions in this system, and our novel network approach can provide general insights into transmission in other multi-host disease systems.
\end{abstract}




\section{Keywords}

European badger, cattle, bovine tuberculosis, Mycobacterium bovis, environmental transmission, wildlife disease, reservoir.

\section{Introduction}

Between-species transmission of infection in multi-host disease systems remains poorly understood, despite representing a potentially important opportunity for disease control (Plowright et al., 2017; Webster et al., 2017). The behaviours that might result in transmission of infections between species are often rare (Viana et al., 2014), with successful transfer and establishment of the pathogen in a new host being rarer still (Woolhouse et al., 2001). As a result, identifying when and where transmission occurs between host species, and the behaviours that make specific individuals or classes important, can be particularly challenging. While advances in pathogen genotyping have helped (Johnston et al., 2010; Mather et al., 2013; Viana et al., 2014; Kamath et al., 2016; Trewby et al., 2016), there is a paucity of evidence on precisely how individual behaviour is associated with opportunities for between-species transmission, even in the best-studied systems.

Pathogen transmission can occur via direct transmission between individuals or indirectly via vectors, fomites or wider environmental sources of infection (McCallum et al., 2001; White et al., 2017). The behaviours that facilitate direct and indirect transmission can be very different; the former requires spatial and temporal co-occurrence and often a specific behavioural interaction, while indirect transmission can simply require spatial co-occurrence within a certain time window (Godfrey, 2013). This distinction can be particularly important when direct interactions among potential hosts are rare, which is likely to be the case for between-species transmission (Viana et al., 2014). Identifying the relative importance of direct and indirect transmission, and the behaviours involved, are therefore likely to be important prerequisites to the development of effective disease management strategies (Plowright et al., 2017; Webster et al., 2017).

The application of network approaches (Silk et al., 2017a,b; White et al., 2017) and movement ecology (Dougherty et al., 2018; White et al., 2018) has become integral to understanding the impact of individual behaviour in disease ecology. Both approaches have exploited the increasing availability of high resolution bio-logging technology to quantify how variation in host behaviour contributes to heterogeneity in pathogen transmission opportunities 
(Krause et al., 2013; White et al., 2017). Recent developments in network analytical approaches have included the development of a general multilayer network approach that considers multiple interdependent networks within the same analytical framework (Kivelä et al., 2014; De Domenico et al., 2016; Pilosof et al., 2017b). This multilayer approach offers new opportunities to consider interactions between host populations (Pilosof et al., 2017a) and to combine social and spatial networks in novel ways (Pilosof et al., 2017b). Here we present a multilayer network as a general framework for quantifying the relative importance of potential routes for direct and indirect pathogen transmission between host species. We apply our framework to an empirical dataset generated using proximity loggers deployed on European badgers Meles meles, domestic cattle Bos taurus and at badger latrines in their shared environment.

Badgers and cattle represent an ideal study system in which to implement this multilayer approach. Both species can be infected by Mycobacterium bovis, the causative agent of bovine tuberculosis (bTB) and in England and Wales this important zoonotic disease costs the government and farmers over $£ 100$ million pounds per year (Godfray et al., 2013; Harris et al., 2017). In badgers, direct transmission via inhalation of aerosolised bacteria (Cheeseman et al., 1989) and, to a lesser extent, biting (Jenkins et al., 2012) are suspected to be key routes for acquiring infection. Hence the distribution of infection in badgers is correlated with social network structure (Weber et al., 2013b). Direct cattle-cattle transmission is also well documented, and thought to occur predominantly via a respiratory route (Goodchild \& CliftonHadley, 2001). Transmission between badgers and cattle, however, remains difficult to trace and quantify. Direct behavioural interactions between badgers and cattle and therefore associated opportunities for direct transmission of M. bovis are rare (Böhm et al., 2009; Drewe et al., 2013; Woodroffe et al., 2016). Given that $M$. bovis can persist in the environment in suitable conditions (Courtenay et al., 2006; King et al., 2015), indirect routes of spread via the environment could play a role in disease transmission between badgers and cattle. In particular, the presence of concentrations of badger excreta at latrine sites on cattle pasture is often identified as a potentially important source of infection for cattle (Williams \& Hoy, 1930; Maddock, 1933; Benham \& Broom, 1991; Courtenay et al., 2006).

Using an empirical dataset derived from a study that quantified badgerbadger, badger-cattle, badger-latrine, cattle-cattle and cattle-latrine con- 
tacts using proximity loggers (Drewe et al., 2013), we constructed a multilayer network of the badger-cattle-environment system. M. bovis is a chronic and slow-spreading infection in badger populations which prevented us from directly investigating transmission over the period when proximity loggers were deployed. However, using a novel multilayer approach we aimed to investigate how direct and indirect contacts may influence transmission opportunities. We predicted that our approach would reveal the potential importance of routes for indirect transmission of infection between badgers and cattle, expecting that indirect contacts via latrines would be integral to connecting the badger and cattle layers of the network. We also predicted that the network positions of badgers within their own social network would reflect their position in the overall multilayer network, as individuals that range further and have more connections would also be expected to use more latrines. Finally, we expected considerable variation among latrines in their potential importance for transmission, depending on how centrally located they were within the study site and the number of badgers from different social groups that used them. We then discuss how this framework might tease apart direct and indirect transmission and help identify individuals that might be particularly important for cross-species transmission.

\section{Methods}

\subsection{Study site}

The data used in this study were collected at Woodchester Park, Gloucestershire, UK (Figure 1; 51 ${ }^{\circ} 43^{\prime} \mathrm{N}, 2^{\circ} 16 \mathrm{~W}$ ) between April and September 2010. The study site is a $7 \mathrm{~km}^{2}$ area of the Cotswold escarpment centred on a wooded valley with pockets of cattle pasture on the valley floor and mixed agriculture in the surrounding area (Figure 1). The site is the location of a long-term study of the ecology, epidemiology and demography of a highdensity badger population (21-23 different social groups) naturally infected with M. bovis (Delahay et al., 2013; McDonald et al., 2018). Social group territories within the study area are defined using bait-marking studies during the period of peak territoriality in the spring (see Delahay et al., 2000), and we use these measures to identify social group territories (Figure 1) and define social group membership throughout this study. 


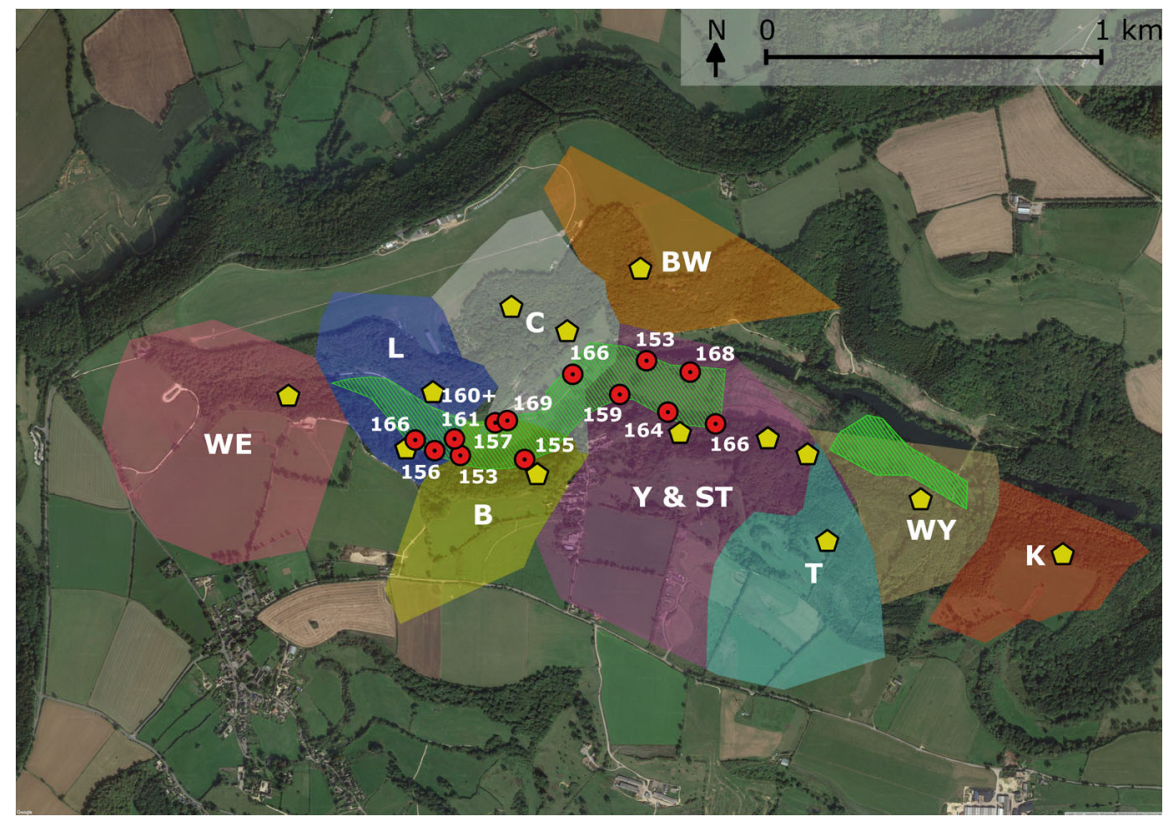

Figure 1. A map of the study site showing badger social group boundaries (coloured polygons), badger main setts (yellow pentagons), the location of active latrines at which base stations were located (red circles), and the areas of cattle pasture (green hatched area). Social group polygons represent the $95 \%$ maximum convex polygon of the, 2010 bait-marking data and are labelled with the identifying codes of the social groups that they contain (see Figure 4). Social group territories can contain multiple main setts.

\subsection{Data collection}

Data were collected on social and spatial behaviour of cattle and badgers using UHF proximity loggers (Sirtrack, Havelock North, New Zealand). These devices operate by transmitting a unique UHF signal and automatically detecting the signal of other loggers that come within a pre-set distance of each other. The end of a contact event is determined when two loggers have stopped detecting one another for a pre-set time period (30 seconds in this study). At this point the date/time and duration of the contact is logged, together with the identity of the encountered logger (Drewe et al., 2012, 2013; Weber et al., 2013b). During the wider study (from September, 2009 until September, 2010), proximity loggers were deployed on a total of 33 Welsh Black cattle, 61 badgers, and for some of the time at 19 base stations located at active badger latrines (Drewe et al., 2013). The analysis presented here focussed only on the period between April and September, 2010, while base 
stations were deployed at latrines and proximity logger data were simultaneously collected from 25 cattle and 42 badgers (either directly through logger downloads or indirectly through interacting with a logger that was subsequently downloaded). This provided contact data for the majority of individual animals in the herd of cattle in the central valley of the study area. Data were temporarily unavailable for some individuals if collar memory banks were full, collars were lost or for the calves in the herd prior to collaring. Contact data was available from approximately $70 \%$ of the adult badgers in the focal social groups in the study area (Drewe et al., 2013; Weber et al., 2013b). Data on latrine use were available from 13 latrines in total (Figure 1).

Badgers were trapped as part of the ongoing long-term study at Woodchester Park (McDonald et al., 2018). Proximity loggers mounted on leather neck collars were fitted to adult badgers (those more than one year old) while they were anaesthetised for routine examination (including ageing and sex determination) and sampling. Cubs cannot be collared for welfare reasons. The detection distance of each badger collar was pre-set to between 0.4 and 1.4 metres (there is some natural variability between collars) to record close contacts that represent potential opportunities for M. bovis transmission. Samples collected while badgers were under anaesthesia were used to ascertain bTB infection status. Badgers were classified as infected if they tested positive for at least one of three diagnostic tests (Drewe et al., 2010): mycobacterial culture of clinical samples (Clifton-Hadley et al., 1993), a serological assay (Stat-Pak; Chambers et al., 2008) and a gammainterferon assay (Dalley et al., 2008) at any capture event prior to when collars were fitted. While these tests individually have their limitations, combining test results increases the likelihood of correctly determining $M$. bovis infection status (Drewe et al., 2010).

Proximity loggers mounted on nylon neck collars were deployed on cattle in the centre of the study area for up to 12 months (the exact duration of collaring varied among individuals). The proximity collars had a detection range of 1.5-1.9 $\mathrm{m}$. The collared cattle during study as a whole comprised 24 females and nine males, 28 of which were adults and five of which were calves born during the study and fitted with proximity loggers in June, 2010. The cattle farm was in an annual testing area for bTB, and so all cattle in the study had tested negative on the tuberculin skin test within the 12 months prior to the start of the study, with the exception of newborn calves. 
Base stations were deployed at active badger latrines in the three main catthe fields in the valley (Figure 1). Base stations were pre-set to detect proximity loggers coming within a horizontal distance at ground-level of $0.3-0.8 \mathrm{~m}$. As this resulted in a greater detection distance above ground-level, data on animal collar logger to base station contact events were extracted from base station datasets (and did not require reciprocal contacts to be recorded by the collar loggers).

\subsection{Social network construction}

Contact data were filtered in the same way as previous studies (Drewe et al., 2012, 2013). Records within a rolling 60 second time interval were amalgamated and then any remaining one second contacts were removed. The filtered data were then used to construct the four constituent networks of a multilayer network framework: (i) a social network of all contacts recorded among badgers, (ii) a spatial bipartite network recording when individual badgers visited latrines monitored by base stations, (iii) a spatial bipartite network recording when individual cattle visited latrines monitored by base stations and (iv) a social network of all contacts among cattle (Figure 2).

\subsection{Network edge definitions}

Edges in each network were weighted by the log of the total duration of contacts within a dyad. Badger-badger edges connected individuals that had come within the detection distance $(0.4-1.4 \mathrm{~m})$ of proximity loggers that were successfully recovered and downloaded. Cattle-cattle edges also connected individuals that had come within the detection distance $(1.4-1.9 \mathrm{~m})$ of proximity loggers that were recovered. Badger-latrine and cattle-latrine edges connected individual animals to latrines if the base station positioned at that latrine recorded a contact. Base stations were pre-set to record contacts within 0.3-0.8 $\mathrm{m}$ at ground level (see Drewe et al, 2013).

\subsection{Social network analysis}

\subsubsection{Position of badgers in within-species and multilayer networks}

The roles of individual badgers within the badger social network and within the overall multilayer network were compared using a range of centrality measures for single and multilayer networks. Multilayer centrality measures for each badger were calculated by transforming the multilayer edge list into a supra-adjacency matrix containing all four networks (Kivelä et al., 2014) 


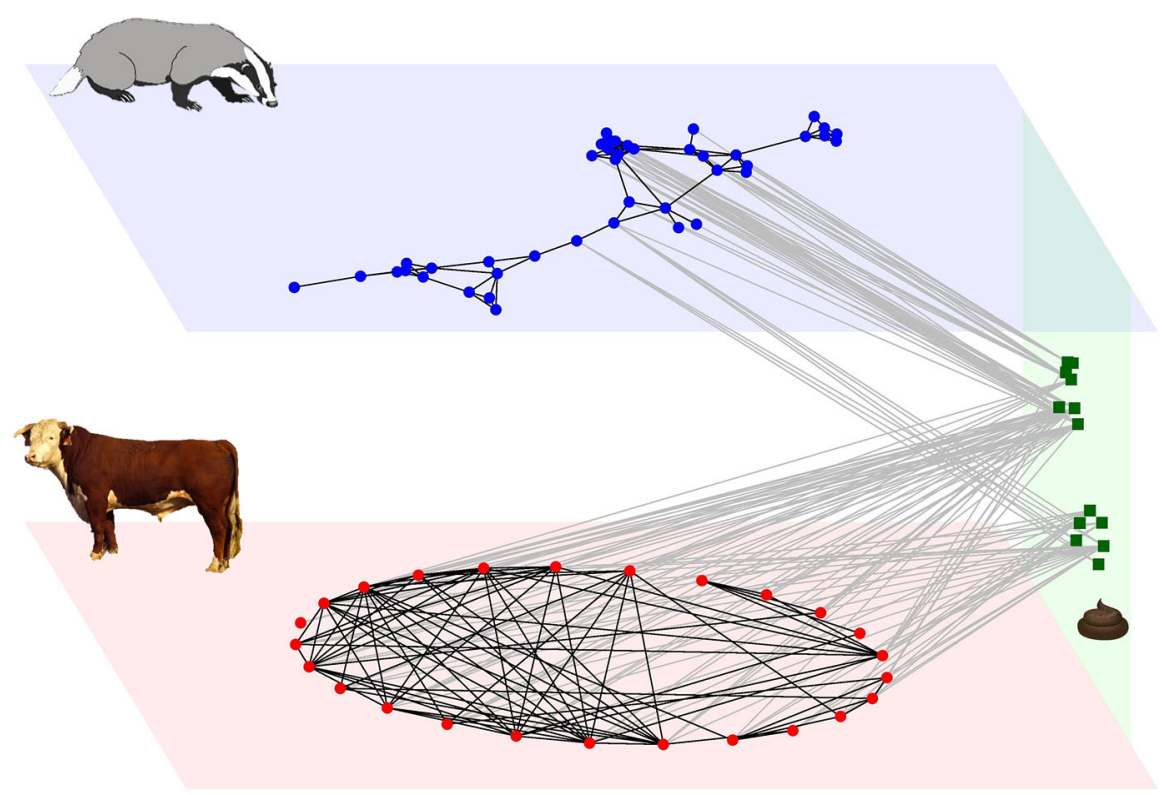

Figure 2. A multilayer contact network for European badgers and domestic cattle at the Woodchester Park study site. The top (blue layer) depicts the badger social network for the entire study period. The bottom (red) layer depicts the cattle social network for the entire study period, with edges filtered for clarity to show only the $25 \%$ of the strongest edges. Locations of nodes in the badger network were determined by layout.auto() in the R package igraph and the locations in the cattle network were determined by the layout_in_circle(). The side (green) layer represents badger latrines (arranged on a plane in correspondence with their location within the study site). Grey edges represent contacts between either badgers or cattle with base stations positioned at those latrines. For each of these bipartite, spatial networks (badger-latrine and cattle-latrine) edges are filtered for clarity to show only the $25 \%$ of the strongest edges.

(Figure A1 in the Appendix) and then analysing the resultant network in the R package igraph (Csardi \& Nepusz, 2006). We calculated four measures of centrality: unweighted degree, weighted degree (strength), eigenvector centrality and betweenness centrality. These measures were selected to provide a spectrum from those measuring purely direct connections (unweighted degree/strength), through accounting for localised indirect connections (eigenvector centrality) to global measures of network position (betweenness centrality). Betweenness was calculated using inverted edge weights as igraph treats greater weight as a cost in its shortest path algorithms (Silk et al., 2017a). The equivalent centrality measures were then calculated for the badger social network only. All measures from each network were scaled by 
dividing by the maximum value for each measure, meaning that values for all centrality measures were between 0 and 1 for each network.

We then tested whether the network position of badgers within the overall multilayer network depended on phenotypic traits using a randomisationbased approach (Farine \& Whitehead, 2015; Silk et al., 2017b). We fitted generalised linear mixed effects models with unweighted degree (logistic transformation of scaled values, Gaussian error distribution), weighted degree (logistic transformation of scaled values, Gaussian error distribution), a binary approximation of eigenvector centrality (whether the scaled eigenvector centrality was greater or less than 0.5 ; binomial error distribution) and betweenness (scaled values, zero-inflated beta distribution) as response variables. Models for unweighted degree, weighted degree and eigenvector centrality were fitted in the R package lme4 (Bates et al., 2015). The model for betweenness was fitted in Stan (Carpenter et al., 2017) using the $\mathrm{R}$ package brms (Burkner, 2017). We included sex (male versus female), age (adult ( $2+$ years old) versus yearling (1-2 years old)), bTB infection status (test-positive versus test-negative) and the number of days an individual was known to be collared as explanatory variables. Age was not included in the model for eigenvector centrality as no yearlings had an eigenvector centrality greater than 0.5 . The number of days an individual was known to be collared was scaled to be mean-centred with unit variance. We included social group membership as a random effect to control for differences between individuals generated by variation in the number of individuals collared per group. Badger social group membership was assigned according to which sett an individual was most recently captured at, using bait-marking data from, 2009, 2010 and 2011 to define social group territories (Delahay et al., 2000). Using this combined (multi-year) approach differed from using the 2010 bait marking data only by placing setts $\mathrm{Y}$ and ST as different social group territories, rather than including them as part of the same group. We tested the statistical significance of our model estimates using node-based permutations of the data. We randomly resampled the identity of all individuals in the population 10000 times and recalculated model estimates for these randomised datasets. Terms were considered to be statistically significant in the real dataset if the effect size lay outside the $95 \%$ confidence interval of effect sizes in the randomised datasets. Social group was considered to be more important than expected by chance if the estimate of the variance explained by social group lay outside the $95 \%$ confidence intervals of the equivalent value calculated from the randomised networks. The $p$ value represents the proportion of es- 
timates of the random effect variance from models in randomised networks that were greater than the equivalent estimate from the observed network.

\subsubsection{The role of indirect contacts in disease transmission}

We developed a novel approach to identify the relative importance of indirect contacts via latrines (potential environmental routes for between and withinspecies transmission) that calculated the proportion of all possible shortest paths between pairs of nodes within the unweighted multilayer network that included a latrine. If indirect contacts are vital to the connection of layers (or for connections within layers) then this value would be expected to equal 1 , and if indirect contacts do not ever shorten possible transmission routes between hosts then this value would be expected to be 0 (Figure 3). When both indirect and direct contacts occur, this measure can quantify the extent to which indirect routes can shorten potential transmission pathways. We used unweighted edges to reflect the importance of the existence of these connections due to uncertainty over how weight should be incorporated into epidemiologically relevant shortest path calculations.

We also quantified a similar measure of the importance of indirect contacts via other network layers for network paths between all pairs of individual badgers (dyads) that were collared contemporaneously for a minimum of 10 days. First, we calculated the proportion of the set of shortest paths between badgers that included a latrine for all dyads (817 in total). We then determined the proportion of shortest paths between dyads containing individuals from different groups (711 in total) which included a latrine. This makes it possible to consider the potential relative importance of indirect contacts for within- and between-group transmission (see Figure A2 in the Appendix). The statistical significance of the difference in the proportion of between- versus within-group shortest paths that passed through latrines was assessed by randomly sampling from all possible shortest paths between dyads 100000 times and calculating the equivalent proportion of betweenversus within-group dyads they contained. A similar approach was used to determine the statistical significance of the proportion of between-group dyads that contained a latrine.

\subsubsection{Heterogeneity in importance among latrines}

The potential importance of different latrines in facilitating disease transmission was assessed using multilayer centrality measures. Similar to above, centrality measures were calculated for each latrine from a supra-adjacency 


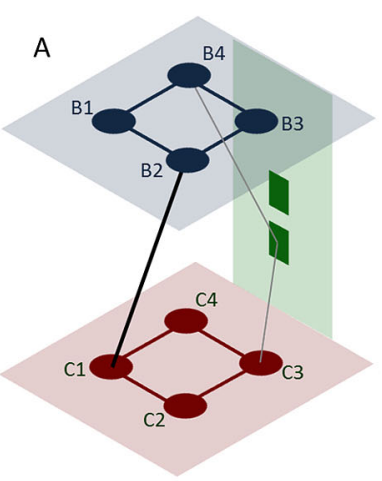

$\mathrm{IP}=\mathbf{0 . 3 1 2 5}$

\begin{tabular}{|c|c|c|c|c} 
& C1 & C2 & C3 & C4 \\
\hline B1 & 0 & 0 & 1 & 0 \\
\hline B2 & 0 & 0 & 0 & 0 \\
\hline B3 & 0 & 0 & 1 & 0 \\
\hline B4 & 0 & 1 & 1 & 1
\end{tabular}

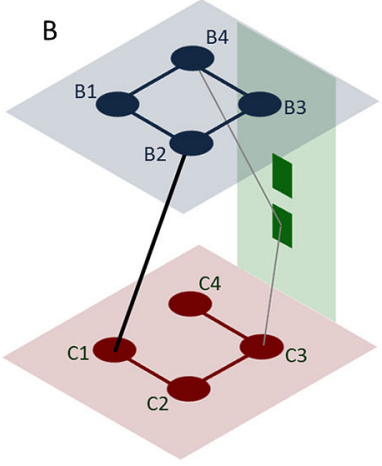

$\mathrm{IP}=\mathbf{0 . 4 3 7 5}$

\begin{tabular}{c|c|c|c|c} 
& C1 & C2 & C3 & C4 \\
\hline B1 & 0 & 0 & 1 & 1 \\
\hline B2 & 0 & 0 & 0 & 0 \\
\hline B3 & 0 & 0 & 1 & 1 \\
\hline B4 & 0 & 1 & 1 & 1
\end{tabular}

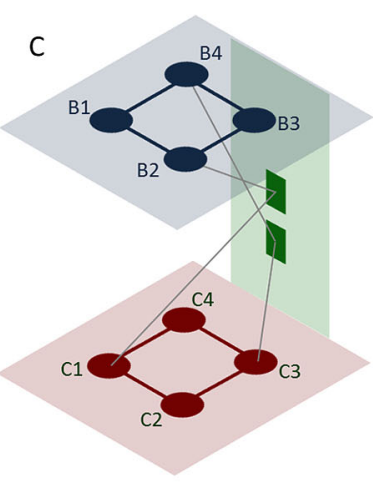

IP $=1$

\section{\begin{tabular}{l|l|l|l|} 
C1 & C2 & C3 & C4 \\
\hline
\end{tabular}}

\begin{tabular}{l|l|l|l|l} 
B1 & 1 & 1 & 1 & 1 \\
\hline B2 & 1 & 1 & 1 & 1 \\
\hline B3 & 1 & 1 & 1 & 1 \\
\hline B4 & 1 & 1 & 1 & 1
\end{tabular}

Figure 3. A graphical depiction of calculating the proportion of inter-layer shortest paths that are indirect (IP). All pairwise combinations in the top ("B") layer and bottom ("C") layer are given a score of 0 if the shortest path through the network between them does not include an indirect/environmental connection, 1 if it does, and 0.5 if the shortest direct and indirect paths are equally short. We illustrate three toy examples where the vast majority of shortest paths are direct (A), direct and indirect routes are similarly important (B), and all shortest paths are indirect $(\mathrm{C})$.

matrix of the multilayer network using the package igraph (Csardi \& Nepusz, 2006). We calculated degree, strength, eigenvector centrality and betweenness centrality to measure the influence and connectivity of each latrine in the full network. Again, betweenness was calculated using inverted edge weights. We additionally calculated the degree and strength of latrines to badgers and cattle separately to determine whether connections to one species or the other were more important in making latrines central within the multilayer network.

We then compared the distribution of the network measures calculated in the observed network to the same distribution calculated from 100000 multilayer networks in which the badger-latrine and cattle-latrine networks had been randomised. These randomisations were restricted so that: (a) cattlelatrine edges could only be swapped to latrines in the same field, but could be swapped to any other cow, and (b) badger-latrine edges could only be 
swapped to another latrine in the same or a neighbouring social group territory or only to another badger from the same social group as the original. Our algorithm for edge swaps: (1) selected an edge to be changed, (2) randomly selected either the animal or the latrine to be swapped, then (3) completed the swap according to the rule outlined above. After every 10 swaps (1000 000 million swaps were conducted in total) all measures were calculated as described above. Both cattle-latrine and badger-latrine edges were randomised (with the order of the swaps occurring randomly in each step of the algorithm).

\section{Results}

\subsection{Position of individual badgers in within-species and multilayer networks}

Individual badgers tended to occupy similar positions in the badger social network as in the multilayer network that also contained badger latrines and cattle interactions (Figures 2 and 4), and this pattern was consistent regardless of the nature of the centrality measure used. The sex, age and infection status of badgers did not generally significantly relate to variation in network centrality of individuals within the overall multilayer network (Table 1). No yearlings were recorded to have high scores for eigenvector centrality. The only additional significant result was for males to be less likely to have high eigenvector centrality scores than females. However, given the considerable standard error around the model predictions in the observed network, this result should be treated with caution. For all measures of network centrality, a considerable amount of variation was explained by the social group affiliation of individual badgers (eigenvector centrality: $p<0.001$, degree: $p=0.004$, strength: $p=0.002$, betweenness: $p<0.001$ ).

\subsection{The role of indirect contacts in disease transmission}

In the wider study by Drewe et al. (2013) a very small number of badgercattle contacts were detected, but in the subset of data used for the present study, no such interactions were represented. All of the shortest paths between the badgers and cattle within the multilayer network included a latrine, indicating the likely importance of indirect contacts for disease transmission in this system. For badger-badger transmission routes, 299 of the 817 possible shortest paths between individuals that were contemporaneously 


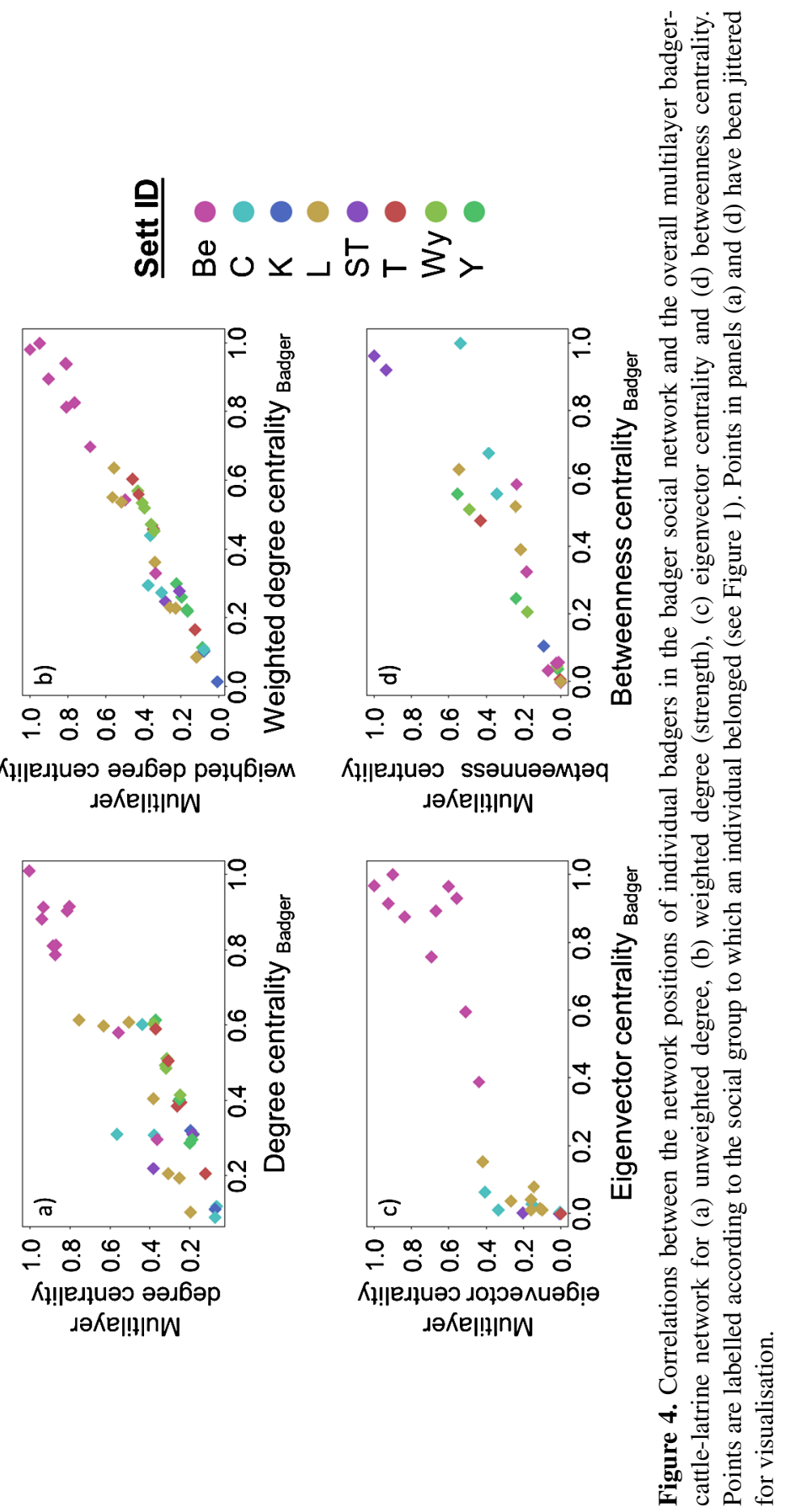


Table 1.

The effect of badger phenotypic characteristics (sex, age, bTB infection status) and the duration of collaring, on network position in the overall multilayer network.

\begin{tabular}{lccccc}
\hline $\begin{array}{l}\text { Centrality } \\
\text { measure }\end{array}$ & Effect & Model Estimate & $\begin{array}{c}2.5 \% \text { quantile } \\
\text { from null } \\
\text { models }\end{array}$ & $\begin{array}{c}\text { 97.5\% quantile } \\
\text { from null } \\
\text { models }\end{array}$ & $p$ \\
\hline Degree & Sex (M vs. F) & $-0.29( \pm 0.31)$ & -0.90 & 0.89 & 0.065 \\
& Infection status & $-0.15( \pm 0.35)$ & -0.90 & 0.91 & 0.789 \\
& $(+$ vs. -$)$ & & & & \\
& Collar duration & $0.20( \pm 0.14)$ & -0.45 & 0.42 & 0.320 \\
Strength & Age (Y vs. A) & $-0.14( \pm 0.42)$ & -1.12 & 1.23 & 0.869 \\
& Sex (M vs. F) & $-0.16( \pm 0.31)$ & -0.90 & 0.89 & 0.260 \\
& Infection status & $-0.31( \pm 0.34)$ & -0.91 & 0.91 & 0.387 \\
& $(+$ vs. -$)$ & & & & \\
Betweennes & Collar duration & $0.19( \pm 0.14)$ & -0.43 & 0.41 & 0.814 \\
& Age (Y vs. A) & $-0.13( \pm 0.41)$ & -1.10 & 1.22 & 0.428 \\
& Sex (M vs. F) & $-1.08( \pm 0.64)$ & -1.40 & 1.45 & 0.359 \\
& Infection status & $0.62( \pm 0.84)$ & -1.43 & 1.63 & 0.262 \\
& $(+$ vs. -$)$ & & & & \\
& Collar duration & $-0.12( \pm 0.27)$ & -0.73 & 0.66 & 0.797 \\
& Age (Y vs. A) & $1.08( \pm 1.03)$ & -1.83 & 2.22 & 0.428 \\
Eigenvector & Sex (M vs. F) & $-3.50( \pm 4.65)$ & -1.91 & 2.22 & $0.002^{*}$ \\
centrality & Infection status & $1.88( \pm 4.31)$ & -2.23 & 1.88 & $0.975^{+}$ \\
& $(+$vs. -$)$ & & & & \\
& Collar duration & $0.10( \pm 1.59)$ & -0.84 & 1.40 & 0.572 \\
& Age (Y vs. A) & NA & NA & NA & NA \\
\hline
\end{tabular}

Model estimates are provided along with the quantiles from equivalent models fitted following node-based permutations of the network. Contrasts are for male vs. female (M vs. F), bTB positive vs. bTB negative (+ vs. - ) and yearling vs. adult ( $\mathrm{Y}$ vs. A). The one-tailed $p$ value is the proportion of estimates from the randomised data that are less than or equal to the model estimate for the observed data and should be considered as statistically significant when less than 0.025 or greater than 0.975 . Results marked * are statistically significant using this permutation test. Results marked ${ }^{+}$are marginally non-significant.

collared for 10 days or more included a latrine. All (100\%) of these 299 shortest paths containing latrines also connected badgers from different territorial social groups, significantly more than would be expected by random chance $(p<0.001,97.5 \%$ quantile of randomisations $=269(90.0 \%))$. These shortest paths via latrines represented 42\% (299/711) of the total number of between-social group shortest paths, which was significantly more than would be expected by chance alone ( $p<0.001,97.5 \%$ quantile of randomisations $=37.8 \%(269 / 711))$. Together, this highlights that indirect 
Table 2.

The observed strength and betweenness centrality (both scaled to be between 0 and 1) of badger latrines in a multilayer contact network between badgers and cattle.

\begin{tabular}{lcccc}
\hline $\begin{array}{l}\text { Badger } \\
\text { latrine }\end{array}$ & $\begin{array}{c}\text { Observed } \\
\text { strength }\end{array}$ & $\begin{array}{c}\text { Expected strength } \\
\text { in randomised } \\
\text { networks }\end{array}$ & $\begin{array}{c}\text { Observed } \\
\text { betweenness } \\
\text { centrality }\end{array}$ & $\begin{array}{c}\text { Expected betweenness } \\
\text { centrality in } \\
\text { randomised networks }\end{array}$ \\
\hline 153 & $0.65>$ & $0.36-0.65$ & $1.00>$ & $0.004-0.983$ \\
154 & $0.44 \sim$ & $0.36-0.65$ & $0.00<$ & $0.002-0.929$ \\
155 & $0.90>$ & $0.36-0.65$ & $0.22 \sim$ & $0.002-0.961$ \\
156 & $0.38 \sim$ & $0.35-0.66$ & $0.90 \sim$ & $0.002-0.954$ \\
157 & $0.88>$ & $0.36-0.65$ & $0.04 \sim$ & $0.002-0.971$ \\
158 & $0.39 \sim$ & $0.36-0.66$ & $0.00<$ & $0.002-0.973$ \\
159 & $0.39 \sim$ & $0.35-0.65$ & $0.00<$ & $0.005-0.961$ \\
160 & $0.47 \sim$ & $0.36-0.65$ & $0.12 \sim$ & $0.005-0.920$ \\
161 & $0.50 \sim$ & $0.36-0.65$ & $0.01 \sim$ & $0.005-0.956$ \\
164 & $0.18<$ & $0.36-0.65$ & $0.00<$ & $0.005-0.944$ \\
166 & $0.17<$ & $0.35-0.65$ & $0.00<$ & $0.002-0.908$ \\
168 & $0.18<$ & $0.36-0.66$ & $0.00<$ & $0.005-0.937$ \\
169 & $1.00>$ & $0.36-0.65$ & $0.99>$ & $0.007-0.939$ \\
\hline
\end{tabular}

Values indicted by $>$ represent an observed centrality measure that is greater than expected by the randomisation of networks, and $<$ represents an observed centrality measure that is lower than expected.

contacts via latrine use are potentially important in providing transmission routes between badger social groups.

\subsection{Heterogeneity in importance among latrines}

Latrines varied considerably in their centrality (see Table 2 and Table A1 in the Appendix), with some being more central or less central in the network than expected by chance. In the observed network both strength (direct connections) and betweenness centrality (global centrality) were dominated by particular latrines to a greater extent than in the randomised networks (Table 2). However, the identity of these latrines differed depending on the centrality measure used (Table 2 and Table A1), especially for betweenness relative to more local centrality measures. The most central latrines tended to have more and stronger connections to both badgers and cattle (Figure A3 in the Appendix). One latrine (node 169) was consistently much more central than expected by chance regardless of the centrality measure used and therefore occupied a particularly important position in the network. 


\section{Discussion}

We have presented a novel multilayer framework for considering the potential role of social and spatial behaviour in pathogen transmission in multihost systems. Using this framework we have demonstrated the potential importance of indirect contacts in providing opportunities for the transmission of $M$. bovis between badgers and cattle. Furthermore, using a network approach has enabled us to reveal important variation among badger latrines in their role in both badger-badger contacts and badger-cattle contacts. Finally, we revealed that the relationship between the role of badgers in the badger social network and the overall multilayer network was similar, indicating that measures of intra-specific social interactions in badger populations can provide a useful proxy for the potential role of individuals in between-species transmission.

Our results are consistent with previous studies which also found that opportunities for direct transmission of $M$. bovis from badgers to cattle at pasture are likely to be rare (Böhm et al., 2009; Drewe et al., 2013; Woodroffe et al., 2016). In addition, our approach enabled us to quantify the role of individual badger latrines within a broader network of direct and indirect transmission opportunities. We revealed considerable variation among latrines in their role in connecting the multilayer network. The most influential latrines were clustered towards the centre of the study site, suggesting that this was partially driven by edge effects, and incomplete coverage of latrines by base stations. However, there were multiple latrines with base stations located within this area of the study site and it was notable that two of those identified as more central (node IDs 157 and 169) occurred in a region of overlap between three distinct badger social group territories. Latrines are likely to be particularly influential or to have high betweenness in the network if they are either contacted by many individuals of both species or connect individuals that are otherwise unlikely to interact. The fact that boundary latrines (i.e., those located at the boundary between different social group territories, such as node IDs 157 and 169 in the present study) are more likely to be visited by badgers from multiple setts is well established (Roper et al., 1993; Delahay et al., 2007). Our analysis reveals the true importance of this to the overall network structure; boundary latrines are responsible for almost half of the shortest paths through the network between pairs of badgers from different groups. However, to be influential within the full multilayer network these latrines must also be frequently visited by cattle. This is a combined 
result of the intensity of use of pasture, the precise location of the latrine and the behaviour of the cattle when they are in the field. Most cattle tend to avoid badger latrines while foraging (Benham \& Broom, 1991), but this avoidance is reduced when overall sward quality is low and as a result of cattle tending to forage closer to field edges, where badger latrines can also be concentrated (Hutchings \& Harris, 1997).

While networks have been used to reveal the importance of heterogeneity in social contacts (VanderWaal \& Ezenwa, 2016; White et al., 2017) and in spatial networks of animal movements (Kao et al., 2006; Keeling et al., 2010; Jacoby \& Freeman, 2016), there has been little empirical research quantifying variation in indirect transmission opportunities. This is important as quantifying and characterising this variation might facilitate the targeting of management efforts at key sources of environmental transmission. For example, further use of multilayer network approaches to study direct and indirect contacts at a greater number of latrines would make it possible to more accurately characterise those that are potentially important for transmission between badger social groups and on to cattle.

Our multilayer network analytical approach enabled us to investigate the extent to which the role of badgers within their own social network correlated with their position in a broader badger-cattle-environment network. There was clear correspondence between the role of individual badgers in the badger social network with their position in the overall network for both direct/local and indirect/global measures of network position. It might be possible that these results are driven by the selection of latrine sites at which base stations were deployed, which tended to be towards the centre of the study site. This could potentially enhance any edge effects that result in badgers in social groups closer to the centre of the study site having higher centrality in the network We know from previous work that badgers that are test positive to $M$. bovis are more likely to occupy more connected roles within the badger social network (Weber et al., 2013b), and to use outlier setts away from the main communal burrow system more frequently at certain times of year (Weber et al., 2013a). These individuals could therefore act as "spread capacitors" that regulate the spread of infection in the badger population (Weber et al., 2013b; Silk et al., 2017a). Given that network position within the badger social network is broadly reflective of the position of individuals in the full multilayer network, it seems likely that these individuals may also be integral in regulating transmission between hosts via the 
environment. Network modelling of this system that takes into account the full multi-host system and varies the relative importance of environmental transmission could reveal the conditions required for heterogeneity in badger social behaviour to influence disease dynamics more generally.

Our results do not provide evidence that might make it possible to predict whether particular individuals are likely to be important for between-species transmission based on non-behavioural traits. There was no clear correlation between age, sex or bTB infection status with any measures of centrality in the overall multilayer network. However, in badger social networks a more in-depth analysis was required to characterise the relationship between network position and infection (Weber et al., 2013b), and therefore it may be that more detailed datasets on badger-latrine-cattle interactions could provide greater insights. There is considerable literature on temporal and individual variation in latrine use by badgers (Roper et al., 1993; Stewart et al., 2002; Delahay et al., 2007), but less information on how and why cattle interact with latrines (but see Benham \& Broom, 1991; Hutchings \& Harris, 1997). However, it is only by taking the two in combination that the full picture can be revealed, and the use of temporally dynamic methods is likely to be especially illuminating due to the time-lagged nature of transmission. This re-emphasises the importance of identifying the traits that result in individuals occupying these epidemiologically important roles in the contact network (VanderWaal \& Ezenwa, 2016; McDonald et al., 2018), but again highlights the likely importance of considering the influence of individual variation in behaviour on network position within the full multi-host system rather than a single component of it.

More generally, our multilayer method provides a useful tool with which to disentangle the potential importance of direct and indirect transmission between different hosts. In particular, quantifying the proportion of shortest paths between layers that pass through environmental reservoirs of infection provides a very clear indication of the impact they may have on multi-host disease dynamics. While, our case study is dominated by indirect transmission, this approach is likely to be especially beneficial in contexts when it is difficult to tease apart differences between direct and indirect transmission routes. The power of this approach in revealing how indirect contacts affect the network position of individual badgers is a good illustration of this. Clearly, our approach will be most applicable when there are spatiallydiscrete peaks in indirect/environmental transmission risk (the latrines in our 
study system being a good example). However, while this may be somewhat limiting, there are numerous other examples where such peaks in environmental transmission might occur from shared refuge use (Godfrey et al., 2009; Leu et al., 2010) through to resource patches such as watering holes (Paull et al., 2012; Barasona et al., 2014) or anthropogenic food sources (Becker et al., 2015). In addition, the importance of indirect transmission will depend on other aspects of the system, such as the ability of the pathogen to persist in the environment and the relative likelihood of transmission occurring via different routes (Webster et al., 2017). However, if this additional information is available then it could be incorporated into a multilayer network model of infection within multi-host systems. By building network models that explicitly incorporate spatial and social layers of multiple hosts it is likely to be possible to make more holistic predictions about dynamics in these multi-host systems that relate to both social behaviour and movement ecology.

\section{Acknowledgements}

Data collection was funded by Defra. MJS is funded by a Natural Environment Research Council grant (NE/M004546/1) awarded to RAM, DJH, DPC, MB and RJD. The authors would like to thank the field team at Woodchester Park for assistance with data collection and Heather O'Connor for help with processing the original dataset, and Stephan Leu and two anonymous reviewers for constructive feedback that improved our revisions.

\section{References}

Barasona, J.A., Latham, M.C., Acevedo, P., Armenteros, J.A., Latham, A.D.M., Gortazar, C., Carro, F., Soriguer, R.C. \& Vicente, J. (2014). Spatiotemporal interactions between wild boar and cattle: implications for cross-species disease transmission. - Vet. Res. 45: 122.

Bates, D., Maechler, M., Bolker, B. \& Walker, S. (2015). Fitting linear mixed-effects models using lme4. - J. Stat. Softw. 67: 1-48.

Becker, D.J., Streicker, D.G. \& Altizer, S. (2015). Linking anthropogenic resources to wildlife-pathogen dynamics: a review and meta-analysis. - Ecol. Lett. 18: 483-495.

Benham, P.F.J. \& Broom, D.M. (1991). Responses of dairy cows to badger urine and faeces on pasture with reference to bovine tuberculosis transmission. — Br. Vet. J. 147: 517-532.

Böhm, M., Hutchings, M.R. \& White, P.C.L. (2009). Contact networks in a wildlife-livestock host community: identifying high-risk individuals in the transmission of bovine TB among badgers and cattle. - PLoS One 4: e5016. 
Burkner, P.-C. (2017). Brms: an R package for Bayesian multilevel models using Stan. J. Stat. Softw. 80: 1-28.

Carpenter, B., Gelman, A., Hoffman, M.D., Lee, D., Goodrich, B., Betancourt, M., Brubaker, M., Guo, J., Li, P. \& Riddell, A. (2017). Stan: a probabilistic programming language. J. Stat. Softw. 76: DOI:10.18637/jss.v076.i01.

Chambers, M.A., Crawshaw, T., Waterhouse, S., Delahay, R., Hewinson, R.G. \& Lyashchenko, K.P. (2008). Validation of the BrockTB Stat-Pak assay for detection of tuberculosis in Eurasian badgers (Meles meles) and influence of disease severity on diagnostic accuracy. - J. Clin. Microbiol. 46: 1498-1500.

Cheeseman, C.L., Wilesmith, J.W. \& Stuart, F.A. (1989). Tuberculosis: the disease and its epidemiology in the badger, a review. — Epidemiol. Infect. 103: 113-125.

Clifton-Hadley, R.S., Wilesmith, J.W. \& Stuart, F.A. (1993). Mycobacterium bovis in the European badger (Meles meles): epidemiological findings in tuberculous badgers from a naturally infected population. - Epidemiol. Infect. 111: 9-19.

Courtenay, O., Reilly, L.A., Sweeney, F.P., Hibberd, V., Bryan, S., Ul-Hassan, A., Newman, C., Macdonald, D.W., Delahay, R.J. \& Wilson, G.J. (2006). Is Mycobacterium bovis in the environment important for the persistence of bovine tuberculosis? - Biol. Lett. 2: 460-462.

Csardi, G. \& Nepusz, T. (2006). The igraph software package for complex network research. — Int. J. Compl. Syst. 1695: 1-9.

Dalley, D., Davé, D., Lesellier, S., Palmer, S., Crawshaw, T., Hewinson, R.G. \& Chambers, M. (2008). Development and evaluation of a gamma-interferon assay for tuberculosis in badgers (Meles meles). — Tuberculosis 88: 235-243.

De Domenico, M., Granell, C., Porter, M.A. \& Arenas, A. (2016). The physics of spreading processes in multilayer networks. — Nat. Phys. 12: 901-906.

Delahay, R.J., Brown, J.A., Mallinson, P.J., Spyvee, P.D., Handoll, D., Rogers, L.M. \& Cheeseman, C.L. (2000). The use of marked bait in studies of the territorial organization of the European badger (Meles meles). — Mamm. Rev. 30: 73-87.

Delahay, R.J., Ward, A.I., Walker, N., Long, B. \& Cheeseman, C.L. (2007). Distribution of badger latrines in a high-density population: habitat selection and implications for the transmission of bovine tuberculosis to cattle. - J. Zool. 272: 311-320.

Delahay, R.J., Walker, N., Smith, G.S., Wilkinson, D., Clifton-Hadley, R.S., Cheeseman, C.L., Tomlinson, A.J. \& Chambers, M.A. (2013). Long-term temporal trends and estimated transmission rates for Mycobacterium bovis infection in andisturbed highdensity badger (Meles meles) population. - Epidemiol. Infect. 141: 1445-1456.

Dougherty, E.R., Seidel, D.P., Carlson, C.J., Spiegel, O. \& Getz, W.M. (2018). Going through the motions: incoroporating movement analyses into disease research. - Ecol. Lett. 21: 588-604.

Drewe, J.A., O’Connor, H.M., Weber, N., McDonald, R.A. \& Delahay, R.J. (2013). Patterns of direct and indirect contact between cattle and badgers naturally infected with tuberculosis. — Epidemiol. Infect. 141: 1467-1475.

Drewe, J.A., Tomlinson, A.J., Walker, N.J. \& Delahay, R.J. (2010). Diagnostic accuracy and optimal use of three tests for tuberculosis in live badgers. - PLoS One 5: e11196. 
Drewe, J.A., Weber, N., Carter, S.P., Bearhop, S., Harrison, X.A., Dall, S.R., McDonald, R.A. \& Delahay, R.J. (2012). Performance of proximity loggers in recording intra-and inter-species interactions: a laboratory and field-based validation study. — PloS One 7: e39068.

Farine, D.R. \& Whitehead, H. (2015). Constructing, conducting and interpreting animal social network analysis. - J. Anim. Ecol. 84: 1144-1163.

Godfray, H.C.J., Donnelly, C.A., Kao, R.R., Macdonald, D.W., McDonald, R.A., Petrokofsky, G., Wood, J.L.N., Woodroffe, R., Young, D.B. \& McLean, A.R. (2013). A restatement of the natural science evidence base relevant to the control of bovine tuberculosis in Great Britain. — Proc. Roy. Soc. Lond. B: Biol. Sci. 280: 20131634.

Godfrey, S.S. (2013). Networks and the ecology of parasite transmission: a framework for wildlife parasitology. — Int. J. Parasitol.: Parasit. Wildl. 2: 235-245.

Godfrey, S.S., Bull, C.M., James, R. \& Murray, K. (2009). Network structure and parasite transmission in a group living lizard, the gidgee skink, Egernia stokesii. — Behav. Ecol. Sociobiol. 63: 1045-1056.

Goodchild, A.V. \& Clifton-Hadley, R.S. (2001). Cattle-to-cattle transmission of Mycobacterium bovis. - Tuberculosis 81: 23-41.

Harris, K.A., Brunton, L., Brouwer, A., Garcia, M.P., Gibbens, J.C., Smith, N.H. \& Upton, P.A. (2017). Bovine TB infection status in cattle in Great Britain in: 2015. — Vet. Rec. 180: 170 .

Hutchings, M.R. \& Harris, S. (1997). Effects of farm management practices on cattle grazing behaviour and the potential for transmission of bovine tuberculosis from badgers to cattle. — Vet. J. 153: 149-162.

Jacoby, D.M.P. \& Freeman, R. (2016). Emerging network-based tools in movement ecology. — Trends Ecol. Evol. 31: 301-314.

Jenkins, H.E., Cox, D.R. \& Delahay, R.J. (2012). Direction of association between bite wounds and Mycobacterium bovis infection in badgers: implications for transmission. PLoS One 7: e45584.

Johnston, A.R., Gillespie, T.R., Rwego, I.B., McLachlan, T.L.T., Kent, A.D. \& Goldberg, T.L. (2010). Molecular epidemiology of cross-species Giardia duodenalis transmission in western Uganda. — PLoS Negl. Trop. Dis. 4: e683.

Kamath, P.L., Foster, J.T., Drees, K.P., Luikart, G., Quance, C., Anderson, N.J., Clarke, P.R., Cole, E.K., Drew, M.L. \& Edwards, W.H. (2016). Genomics reveals historic and contemporary transmission dynamics of a bacterial disease among wildlife and livestock. — Nature Commun. 7: 11448.

Kao, R.R., Danon, L., Green, D.M. \& Kiss, I.Z. (2006). Demographic structure and pathogen dynamics on the network of livestock movements in Great Britain. - Proc. Roy. Soc. Lond. B: Biol. Sci. 273: 1999-2007.

Keeling, M.J., Danon, L., Vernon, M.C. \& House, T.A. (2010). Individual identity and movement networks for disease metapopulations. - Proc. Natl. Acad. Sci. USA 107: 88668870 . 
King, H.C., Murphy, A., James, P., Travis, E., Porter, D., Hung, Y.-J., Sawyer, J., Cork, J., Delahay, R.J. \& Gaze, W. (2015). The variability and seasonality of the environmental reservoir of Mycobacterium bovis shed by wild European badgers. - Sci. Rep. 5: 12318.

Kivelä, M., Arenas, A., Barthelemy, M., Gleeson, J.P., Moreno, Y. \& Porter, M.A. (2014). Multilayer networks. - J. Compl. Netw. 2: 203-271.

Krause, J., Krause, S., Arlinghaus, R., Psorakis, I., Roberts, S. \& Rutz, C. (2013). Reality mining of animal social systems. - Trends Ecol. Evol. 28: 541-551.

Leu, S.T., Kappeler, P.M. \& Bull, C.M. (2010). Refuge sharing network predicts ectoparasite load in a lizard. — Behav. Ecol. Sociobiol. 64: 1495-1503.

Maddock, E.C.G. (1933). Studies on the survival time of the bovine tubercle bacillus in soil, soil and dung, in dung and on grass, with experiments on the preliminary treatment of infected organic matter and the cultivation of the organism. - J. Hyg. 33: 103-117.

Mather, A.E., Reid, S.W.J., Maskell, D.J., Parkhill, J., Fookes, M.C., Harris, S.R., Brown, D.J., Coia, J.E., Mulvey, M.R. \& Gilmour, M.W. (2013). Distinguishable epidemics of multidrug-resistant Salmonella typhimurium DT104 in different hosts. - Science 341: 1514-1517.

McCallum, H., Barlow, N. \& Hone, J. (2001). How should pathogen transmission be modelled? — Trends Ecol. Evol. 16: 295-300.

McDonald, J.L., Robertson, A. \& Silk, M.J. (2018). Wildlife disease ecology from the individual to the population: insights from a long-term study of a naturally-infected European badger population. - J. Anim. Ecol. 87: 101-112.

Paull, S.H., Song, S., McClure, K.M., Sackett, L.C., Kilpatrick, A.M. \& Johnson, P.T.J. (2012). From superspreaders to disease hotspots: linking transmission across hosts and space. - Front. Ecol. Environm. 10: 75-82.

Pilosof, S., Greenbaum, G., Krasnov, B.R. \& Zelnik, Y.R. (2017a). Asymmetric disease dynamics in multihost interconnected networks. - J. Theor. Biol. 430: 237-244.

Pilosof, S., Porter, M.A., Pascual, M. \& Kéfi, S. (2017b). The multilayer nature of ecological networks. — Nature Ecol. Evol. 1: 0101.

Plowright, R.K., Parrish, C.R., McCallum, H., Hudson, P.J., Ko, A.I., Graham, A.L. \& LloydSmith, J.O. (2017). Pathways to zoonotic spillover. — Nature Rev. Microbiol. 15: 502510.

Roper, T.J., Conradt, L., Butler, J., Christian, S.E., Ostler, J. \& Schmid, T.K. (1993). Territorial marking with faeces in badgers (Meles meles): a comparison of boundary and hinterland latrine use. - Behaviour 127: 289-307.

Silk, M.J., Croft, D.P., Delahay, R.J., Hodgson, D.J., Boots, M., Weber, N. \& McDonald, R.A. (2017a). Using social network measures in wildlife disease ecology, epidemiology, and management. - BioScience 67: 245-257.

Silk, M.J., Croft, D.P., Delahay, R.J., Hodgson, D.J., Weber, N., Boots, M. \& McDonald, R.A. (2017b). The application of statistical network models in disease research. - Methods Ecol. Evol. 8: 1026-1041.

Stewart, P.D., MacDonald, D.W., Newman, C. \& Tattersall, F.H. (2002). Behavioural mechanisms of information transmission and reception by badgers, Meles meles, at latrines. Anim. Behav. 63: 999-1007. 
Trewby, H., Wright, D., Breadon, E.L., Lycett, S.J., Mallon, T.R., McCormick, C., Johnson, P., Orton, R.J., Allen, A.R. \& Galbraith, J. (2016). Use of bacterial whole-genome sequencing to investigate local persistence and spread in bovine tuberculosis. - Epidemics 14: 26-35.

VanderWaal, K.L. \& Ezenwa, V.O. (2016). Heterogeneity in pathogen transmission: mechanisms and methodology. — Funct. Ecol. 30: 1606-1622.

Viana, M., Mancy, R., Biek, R., Cleaveland, S., Cross, P.C., Lloyd-Smith, J.O. \& Haydon, D.T. (2014). Assembling evidence for identifying reservoirs of infection. - Trends Ecol. Evol. 29: 270-279.

Weber, N., Bearhop, S., Dall, S.R.X., Delahay, R.J., McDonald, R.A. \& Carter, S.P. (2013a). Denning behaviour of the European badger (Meles meles) correlates with bovine tuberculosis infection status. - Behav. Ecol. Sociobiol. 67: 471-479.

Weber, N., Carter, S.P., Dall, S.R.X., Delahay, R.J., McDonald, J.L., Bearhop, S. \& McDonald, R.A. (2013b). Badger social networks correlate with tuberculosis infection. - Curr. Biol. 23: R915-R916.

Webster, J.P., Borlase, A. \& Rudge, J.W. (2017). Who acquires infection from whom and how? Disentangling multi-host and multi-mode transmission dynamics in the "elimination” era. — Phil. Trans. Roy. Soc. Lond. B: Biol. Sci. 372: 20160091.

White, L.A., Forester, J.D. \& Craft, M.E. (2017). Using contact networks to explore mechanisms of parasite transmission in wildlife. - Biol. Rev. 92: 389-409.

White, L.A., Forester, J.D. \& Craft, M.E. (2018). Dynamic, spatial models of parasite transmission in wildlife: their structure, applications, and remaining challenges. - J. Anim. Ecol. 87: 559-580.

Williams, R.S. \& Hoy, W.A. (1930). The viability of B. tuberculosis (Bovinus) on pasture land, in stored faeces and in liquid manure. - Epidemiol. Infect. 30: 413-419.

Woodroffe, R., Donnelly, C.A., Ham, C., Jackson, S.Y.B., Moyes, K., Chapman, K., Stratton, N.G. \& Cartwright, S.J. (2016). Badgers prefer cattle pasture but avoid cattle: implications for bovine tuberculosis control. — Ecol. Lett. 19: 1201-1208.

Woolhouse, M.E.J., Taylor, L.H. \& Haydon, D.T. (2001). Population biology of multihost pathogens. - Science 292: 1109-1112. 


\section{Appendix}

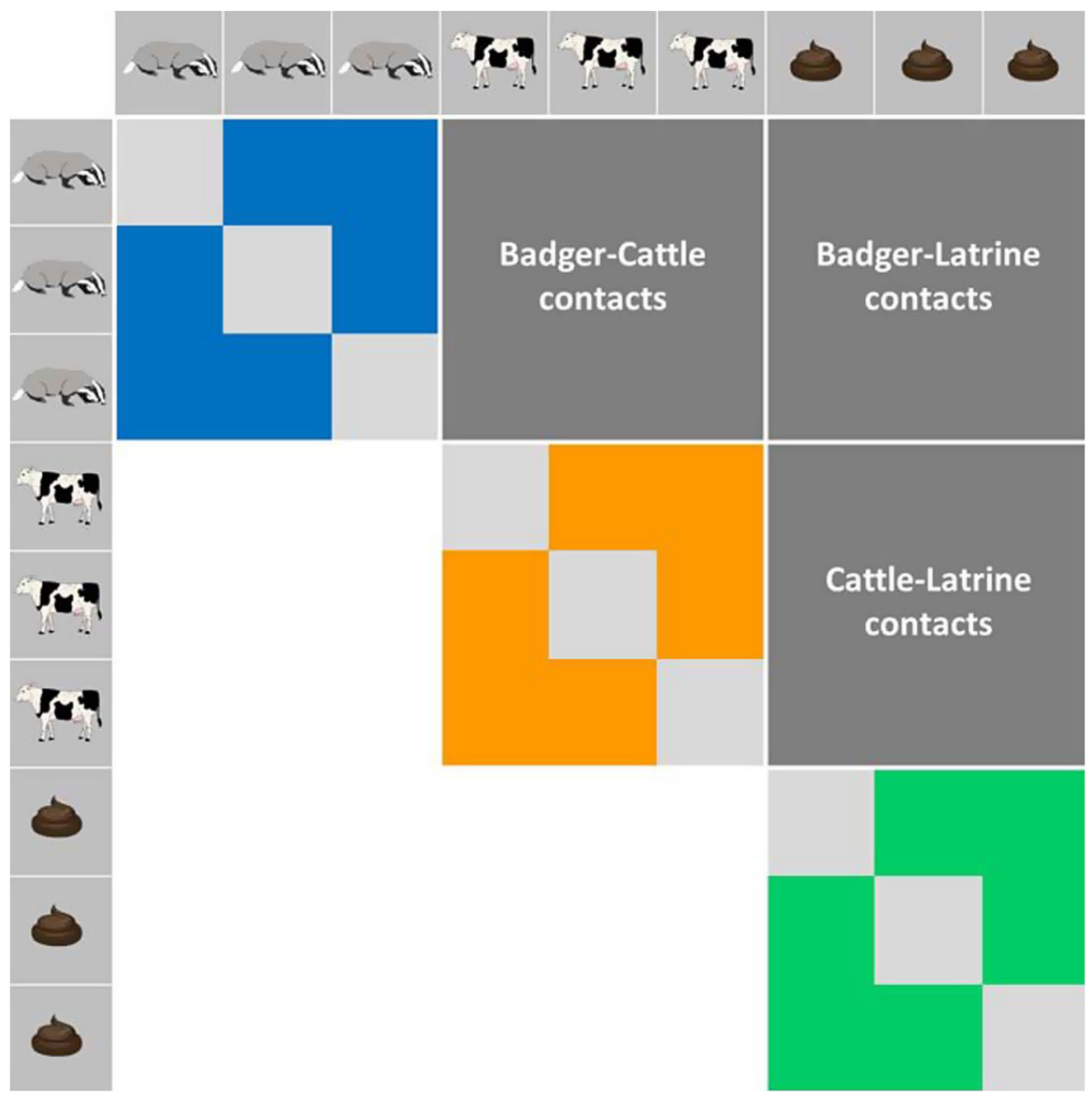

Figure A1. An illustration of the structure of the supra-adjacency matrix used to quantify multilayer social network measures for the multispecies network of potential transmission opportunities. 


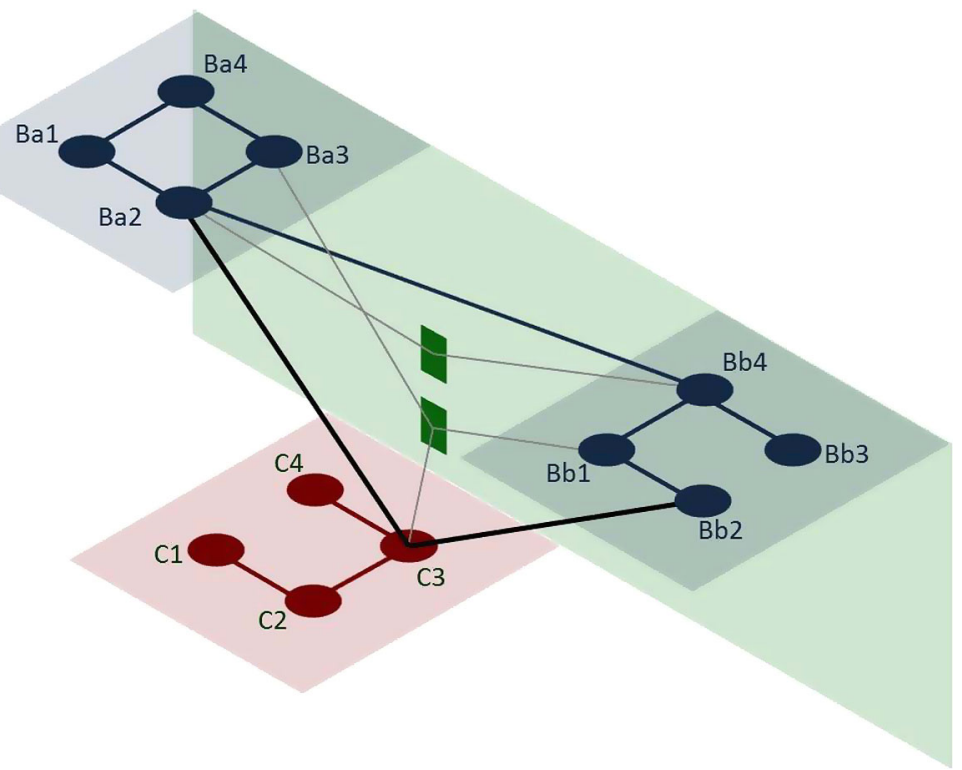

Figure A2. A toy example of the calculation of the proportion of indirect shortest paths (IP) within a single network layer. In this example the shortest paths $\mathrm{Ba} 2-\mathrm{Bb} 4$ and $\mathrm{Ba} 1-\mathrm{Bb} 3$ are direct (i.e., all within the same layer as the nodes being considered) while the shortest paths $\mathrm{Ba} 3-\mathrm{Bb} 1$ and $\mathrm{Ba} 4-\mathrm{Bb} 2$ are indirect as they pass through other network layers. 

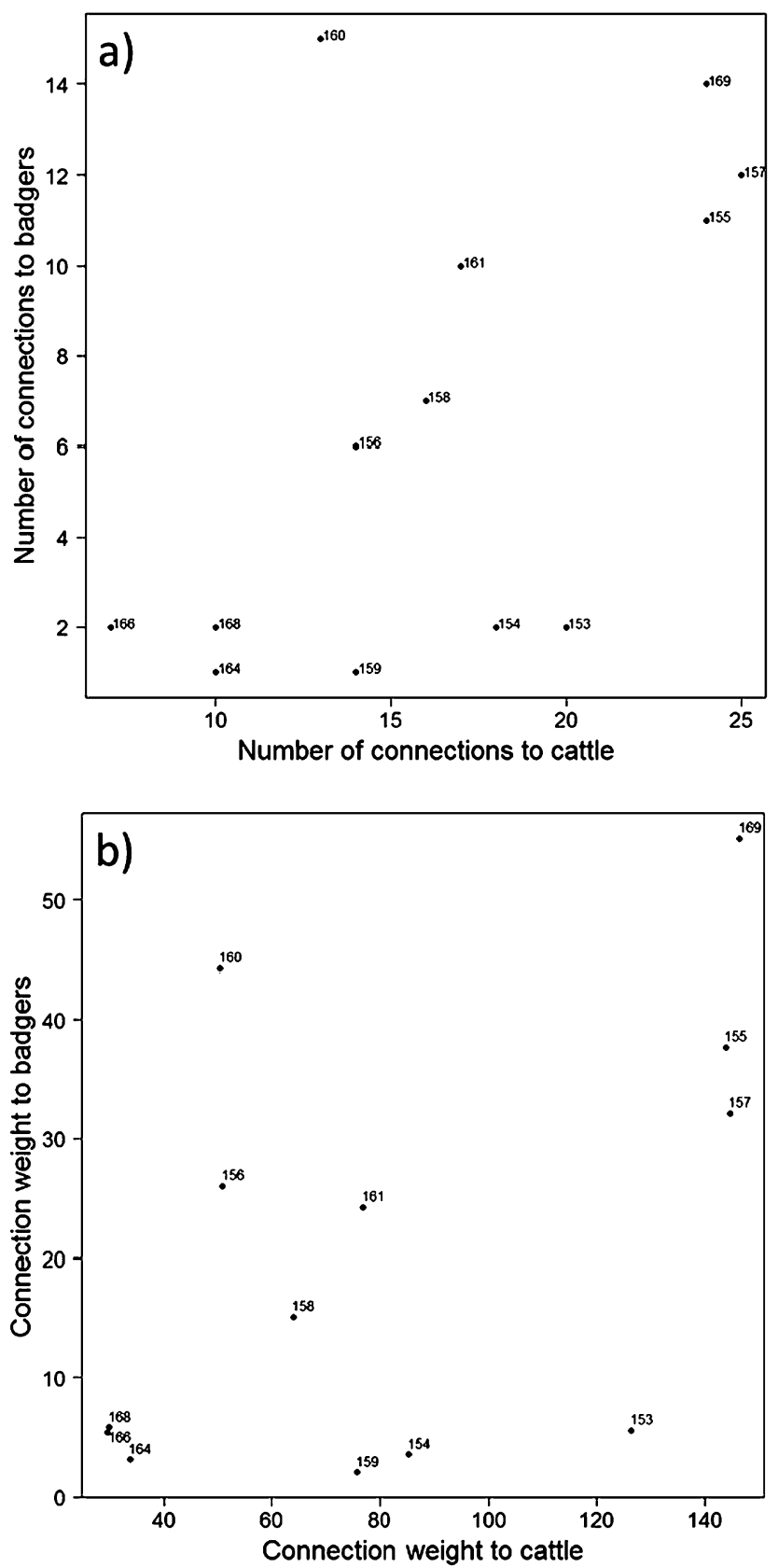

Figure A3. (a) The number of connections and (b) the overall strength of those connections of latrines to the badger layer and cattle layer of the overall multilayer network. 


\section{Table A1.}

The observed degree and eigenvector centrality of badger latrines in a multilayer contact network between badgers and cattle.

\begin{tabular}{lcccc}
\hline $\begin{array}{l}\text { Badger } \\
\text { latrine }\end{array}$ & $\begin{array}{c}\text { Observed } \\
\text { degree }\end{array}$ & $\begin{array}{c}\text { Expected degree } \\
\text { in randomised } \\
\text { networks }\end{array}$ & $\begin{array}{c}\text { Observed } \\
\text { eigenvector } \\
\text { centrality }\end{array}$ & $\begin{array}{c}\text { Expected eigenvector } \\
\text { centrality in } \\
\text { randomised networks }\end{array}$ \\
\hline 153 & $0.58 \sim$ & $0.45-0.76$ & $0.87>$ & $0.38-0.73$ \\
154 & $0.53 \sim$ & $0.45-0.76$ & $0.58 \sim$ & $0.38-0.73$ \\
155 & $0.92>$ & $0.45-0.76$ & $0.96>$ & $0.37-0.73$ \\
156 & $0.53 \sim$ & $0.45-0.76$ & $0.36<$ & $0.37-0.73$ \\
157 & $0.97>$ & $0.45-0.76$ & $0.98>$ & $0.38-0.73$ \\
158 & $0.61 \sim$ & $0.45-0.76$ & $0.46 \sim$ & $0.38-0.74$ \\
159 & $0.39<$ & $0.42-0.76$ & $0.51 \sim$ & $0.38-0.73$ \\
160 & $0.74 \sim$ & $0.45-0.76$ & $0.37<$ & $0.38-0.73$ \\
161 & $0.71 \sim$ & $0.45-0.76$ & $0.54 \sim$ & $0.37-0.73$ \\
164 & $0.29<$ & $0.45-0.76$ & $0.23<$ & $0.38-0.73$ \\
166 & $0.24<$ & $0.45-0.76$ & $0.19<$ & $0.38-0.73$ \\
168 & $0.32<$ & $0.45-0.76$ & $0.20<$ & $0.38-0.73$ \\
169 & $1.00>$ & $0.45-0.76$ & $1.00>$ & $0.38-0.73$ \\
\hline
\end{tabular}

Values indicted by $>$ represent an observed centrality measure that is greater than expected by the randomisation of networks, and $<$ represents an observed centrality measure that is lower than expected. The result marked ${ }^{*}$ is not statistically significant, but is equal to the highest unique value from the randomisations (the upper bound of the measure). 